\title{
Properties and Design of Variable-to-Variable Length Codes
}

\author{
HEINER KIRCHHOFFER, DETLEV MARPE, and HEIKO SCHWARZ, Fraunhofer Institute for
}

Telecommunications, Heinrich-Hertz-Institute

THOMAS WIEGAND, Fraunhofer Institute for Telecommunications, Heinrich-Hertz-Institute, and Technical University of Berlin

For the entropy coding of independent and identically distributed (i.i.d.) binary sources, variable-to-variable length $(\mathrm{V} 2 \mathrm{~V})$ codes are an interesting alternative to arithmetic coding. Such a V2V code translates variable length words of the source into variable length code words by employing two prefix-free codes. In this article, several properties of $\mathrm{V} 2 \mathrm{~V}$ codes are studied, and new concepts are developed. In particular, it is shown that the redundancy of a V2V code cannot be zero for a binary i.i.d. source $\{X\}$ with $0<p_{X}(1)<0.5$. Furthermore, the concept of prime and composite $\mathrm{V} 2 \mathrm{~V}$ codes is proposed, and it is shown why composite V2V codes can be disregarded in the search for particular classes of minimum redundancy codes. Moreover, a canonical representation for V2V codes is proposed, which identifies V2V codes that have the same average code length function. It is shown how these concepts can be employed to greatly reduce the complexity of a search for minimum redundancy (size-limited) V2V codes.

CCS Concepts: • Mathematics of computing $\rightarrow$ Coding theory;

Additional Key Words and Phrases: Entropy coding, Huffman coding, package merge algorithm, variable length coding, V2V coding

\section{ACM Reference format:}

Heiner Kirchhoffer, Detlev Marpe, Heiko Schwarz, and Thomas Wiegand. 2018. Properties and Design of Variable-to-Variable Length Codes. ACM Trans. Multimedia Comput. Commun. Appl. 14, 3, Article 75 (July 2018), 19 pages.

https://doi.org/10.1145/3230653

\section{INTRODUCTION}

Consider the problem of entropy coding an independent and identically distributed (i.i.d.) binary source $\{X\}$ for which the probability mass function (pmf) $p_{X}$ shall be known in encoder and decoder. Furthermore, it shall be assumed that $0<p_{X}(1) \leq 0.5$ holds, because a source with $p_{X}(1)>0.5$ can simply be negated before encoding and after decoding. There exist several wellknown approaches that are capable of encoding realizations of $\{X\}$ close to their self-information. These include, e.g., binary arithmetic coding [19], Huffman coding [11] of fixed-length words of the source, or Tunstall codes [24]. The latter two can be seen as special cases of the more general

Authors' addresses: H. Kirchhoffer, D. Marpe, and H. Schwarz, Fraunhofer Institute for Telecommunications, HeinrichHertz-Institute, Einsteinufer 37, Berlin, 10587, Germany; emails: Heiner.Kirchhoffer@hhi.fraunhofer.de, Detlev.Marpe@ hhi.fraunhofer.de, Heiko.Schwarz@hhi.fraunhofer.de; T. Wiegand, Fraunhofer Institute for Telecommunications, HeinrichHertz-Institute, Einsteinufer 37, Berlin, 10587, Germany, Technical University of Berlin, Faculty of Electrical Engineering and Computer Science, Einsteinufer 17D, Berlin 10587, Germany; email: Thomas.Wiegand@hhi.fraunhofer.de.

Permission to make digital or hard copies of part or all of this work for personal or classroom use is granted without fee provided that copies are not made or distributed for profit or commercial advantage and that copies bear this notice and the full citation on the first page. Copyrights for third-party components of this work must be honored. For all other uses, contact the Owner/Author.

2018 Copyright is held by the owner/author(s). Publication rights licensed to ACM.

ACM 1551-6857/2018/07-ART75 \$15.00

https://doi.org/10.1145/3230653 
concept of so-called variable-to-variable length $(\mathrm{V} 2 \mathrm{~V})$ codes where source words of variable length are translated into code words of variable length. As shown by Freeman in Reference [7], V2V codes with arbitrarily low redundancy can be designed by following a simple construction strategy. However, V2V codes produced in this way turn out to require a rather large number of rather long source and code words to not exceed a certain redundancy value. Freeman exemplifies in Reference [7] that significantly smaller V2V codes (in terms of source and code word lengths and counts) exist for a particular desired redundancy. This observation motivates the search for V2V codes that are more efficient in terms of size and redundancy.

Potential applications for entropy coding of binary i.i.d. sources seem to be rather limited. However, there exists an interesting use case. The so-called probability interval partitioning entropy (PIPE) coding scheme $[16,17,25]$ translates arbitrary sources into a number of binary i.i.d. sources for which appropriate entropy coders are required. These can be implemented as V2V codes.

In this work, several properties of V2V codes are discussed. ${ }^{1}$ In particular, we will formally prove that the redundancy of a V2V code cannot be zero for $0<p_{X}(1)<0.5$. Furthermore, it is observed that a V2V code contributes to its average code length (and thus also to its redundancy) only through the counts of ones and zeros of its source words and through the lengths of associated code words. Based on this, so-called canonical V2V codes are defined, which identify V2V codes that have the same average code length function. A search for codes with minimum average code length can be carried out on a set of canonical V2V codes, which can greatly reduce the computational complexity. Furthermore, it is shown that particular V2V codes can be composed of other V2V codes, which is the motivation for the concept of prime and composite V2V codes. Interestingly, the average code length of a composite V2V code lies between the average code lengths of the prime V2V codes it is composed of. This may be a useful property when searching for $\mathrm{V} 2 \mathrm{~V}$ codes with minimum average code length. Several classes of size-limited V2V codes are discussed, and it is shown how the concepts of canonical, prime, and composite $\mathrm{V} 2 \mathrm{~V}$ codes can be employed to reduce the computational complexity of finding minimum average code length $\mathrm{V} 2 \mathrm{~V}$ codes.

\section{DEFINITIONS AND NOTATION}

Each leaf node of a rooted binary tree can unambiguously be identified by a binary word that describes the path from the root node to the leaf node. A set that contains one such binary word for each leaf node fully describes the rooted binary tree. Such a set shall be called word set of the associated rooted binary tree. For $i \in \mathbb{Z}^{+}$, let $\mathcal{P}_{i}$ be a set containing the word sets of all possible full binary trees ${ }^{2}$ with exactly $i$ leaf nodes. Then

$$
\mathcal{P}=\bigcup_{i \in \mathbb{Z}^{+}} \mathcal{P}_{i+1}
$$

shall be the set of word sets of all possible full binary trees. Note that $\mathcal{P}_{0}=\mathcal{P}_{1}=\emptyset$. A V2V code can be described by two full binary trees, which shall be called source tree and code tree, and a bijective mapping between the leaf nodes of the source tree and the leaf nodes of the code tree. Binary words of the source tree shall be called source words, and binary words of the code tree shall be called code words. The word set of the source tree shall be called source word set, and the word set of the code tree shall be called code word set. Let $\mathcal{S} \in \mathcal{P}_{i}$ be a source word set, and let $C \in \mathcal{P}_{i}$ be a code word set. A bijective mapping between $\mathcal{S}$ and $C$ can be realized through an index permutation function $\pi \in \Pi_{i}$, where $\Pi_{i}$ shall be the set of all possible index permutation functions of $i$ items.

\footnotetext{
${ }^{1}$ The most concepts in this article can also be found in the Ph.D. dissertation [13] of the first author.

${ }^{2} \mathrm{~A}$ full binary tree is a rooted tree where each node has 0 or 2 child nodes.
} 
A V2V code is then fully described through a triple,

$$
(\mathcal{S}, \pi, C) \in \mathcal{V}_{i}
$$

where

$$
\mathcal{V}_{i}=\mathcal{P}_{i} \times \Pi_{i} \times \mathcal{P}_{i}
$$

is the set of all possible $\mathrm{V} 2 \mathrm{~V}$ codes with $i$ source words and $i$ code words. Accordingly, the set of all possible $\mathrm{V} 2 \mathrm{~V}$ codes $\mathcal{V}$ shall be given as

$$
\mathcal{V}=\bigcup_{i \in \mathbb{Z}^{+}} \mathcal{V}_{i+1}
$$

Encoding of a binary i.i.d. source $\{X\}$ with a V2V code $v=(\mathcal{S}, \pi, C)$ corresponds to parsing the source symbols with the source word set $\mathcal{S}$ into a sequence of source words, which shall be modeled as an i.i.d. random process $\{S\}$ over $\mathcal{S}$. Let function $k(\sigma, v)$ yield the code word $c \in C$ that is bijectively mapped to source word $\sigma \in \mathcal{S}$ through the index permutation function $\pi$. By employing function $k$, the source words are translated into code words. The resulting sequence of code words shall be modeled as an i.i.d. random process $\{C\}$ over $C$ and the concatenated code words are the encoded representation. The probability of a source word $\sigma$ is given as pmf value

$$
p_{S}(\sigma)=p_{X}(1)^{M(\sigma)}\left(1-p_{X}(1)\right)^{N(\sigma)},
$$

where $M(x)$ shall be the count of ones and $N(x)$ shall be the count of zeros in a binary word $x$. The average source word length shall be given as

$$
\bar{\ell}(S)=\sum_{\sigma \in \mathcal{S}} p_{S}(\sigma) \ell(\sigma)
$$

with $\ell(x)=M(x)+N(x)$. Jelinek and Schneider prove in Reference [12, Lemma 5] that

$$
H(S)=\bar{\ell}(S) H(X),
$$

where $H(S)$ and $H(X)$ are the source entropies of $S$ and $X$, respectively. The average code word length shall be given as

$$
\bar{\ell}(C)=\sum_{c \in C} p_{C}(c) \ell(c)=\sum_{\sigma \in \mathcal{S}} p_{S}(\sigma) \ell(k(\sigma, v)),
$$

and the average code length shall be given as

$$
\bar{\ell}_{v}(X)=\frac{\bar{\ell}(C)}{\bar{\ell}(S)}=\frac{\sum_{\sigma \in \mathcal{S}} p_{S}(\sigma) \ell(k(\sigma, v))}{\sum_{\sigma \in \mathcal{S}} p_{S}(\sigma) \ell(\sigma)} .
$$

It can be understood as the averaged encoded length per binary input symbol of $X$, encoded with $\mathrm{V} 2 \mathrm{~V}$ code $v$. The average code word length (3) can be written as

$$
\bar{\ell}(C)=H(S)+\sum_{\sigma \in \mathcal{S}} p_{S}(\sigma) \log _{2} \frac{p_{S}(\sigma)}{2^{-\ell(k(\sigma, v))}} .
$$

An i.i.d. random process $\{\hat{S}\}$ with pmf

$$
p_{\hat{S}}(\sigma)=2^{-\ell(k(\sigma, v))}
$$

shall be defined over $\mathcal{S}$ so that Equation (5) can be written as

$$
\bar{\ell}(C)=H(S)+D(S \| \hat{S})
$$


where $D(S \| \hat{S})$ is the Kullback-Leibler divergence [5] of $\hat{S}$ from $S$. According to Reference [5, Theorem 2.6.3], $D(S \| \hat{S}) \geq 0$ is fulfilled with equality if and only if

$$
p_{S}(\sigma)=2^{-\ell(k(\sigma,(\mathcal{S}, \pi, C)))}
$$

holds for all $\sigma \in \mathcal{S}$. The redundancy of $\mathrm{V} 2 \mathrm{~V}$ code $v$ shall be given as

$$
R_{v}(X)=\bar{\ell}_{v}(X)-H(X) .
$$

Substituting Equations (4), (6), and (2) into Equation (8) yields

$$
R_{v}(X)=\frac{D(S \| \hat{S})}{\bar{\ell}(S)} .
$$

Note that there exists a simple rule for finding a (not necessarily unique) $\pi \in \Pi_{i}$ that minimizes the average code length when $\mathcal{S}, C \in \mathcal{P}_{i}$ and $p_{X}(1)$ are given: Simply sort the source words by their probabilities and the code words by their lengths and assign source words with higher probabilities to code words of shorter lengths.

\section{CANONICAL V2V CODES}

A V2V code contributes to its redundancy (8) only through source word probabilities, source word lengths, and associated code word lengths. The source word probabilities only depend on $p_{X}(1)$ and on the counts of ones and zeros of a source word according to Equation (1). As shown in Reference [13, Section 3.6], there exist distinct source trees that do not differ in their source word probability polynomials. For a source word set $\mathcal{S}$, let

$$
\mathrm{W}(\mathcal{S}, X)=\left\{p_{X}(1)^{M(x)}\left(1-p_{X}(1)\right)^{N(x)} \mid x \in \mathcal{S}\right\}_{b}
$$

be the multiset ${ }^{3}$ of source word probability polynomials (1).

Definition 3.1. Two source word sets $\mathcal{S}_{1}$ and $\mathcal{S}_{2}$ are called equivalent if the two associated multisets of source word probability polynomials $\mathrm{W}\left(\mathcal{S}_{1}, X\right)$ and $\mathrm{W}\left(\mathcal{S}_{2}, X\right)$ are identical.

Based on the concept of equivalent source word sets, a canonical representation of source words and source word sets shall be defined.

Definition 3.2. For a source word $\sigma$, the corresponding canonical source word shall be defined as $\mathrm{Cw}(\sigma)=(M(\sigma), N(\sigma))$. In other words, it consists of the number of ones $M(\sigma)$ and the number of zeros $N(\sigma)$ of source word $\sigma$.

Definition 3.3. For a source word set $\mathcal{S}$, the corresponding canonical source word multiset shall be defined as

$$
\operatorname{Cs}(\mathcal{S})=\{\operatorname{Cw}(\sigma) \mid \sigma \in \mathcal{S}\}_{b},
$$

i.e., it consists of the multiset of canonical source words of $\mathcal{S}$.

Lemma 3.4. Two source word sets are equivalent according to Definition 3.1 (i.e., their associated multisets of source word probability polynomials are identical) if and only if their corresponding canonical source word multisets are identical.

Proof. Clearly, if two canonical source word multisets are identical, then they have the same source word polynomials and are thus equivalent. To prove the other direction of the claim, it obviously suffices to show that if two source words $\sigma_{1}$ and $\sigma_{2}$ have the same source word probability

\footnotetext{
${ }^{3}$ Multiset definitions are subscripted with a small $b$ like, e.g., $\{1,1,2\}_{b}$ to distinguish them from set definitions.
} 
polynomials, then one has $\mathrm{Cw}\left(\sigma_{1}\right)=\mathrm{Cw}\left(\sigma_{2}\right)$. The associated source word probability polynomials as defined in Equation (1) are equal if and only if

$$
p_{X}(1)^{M\left(\sigma_{1}\right)} p_{X}(0)^{N\left(\sigma_{1}\right)}=p_{X}(1)^{M\left(\sigma_{2}\right)} p_{X}(0)^{N\left(\sigma_{2}\right)}
$$

holds. Equation (10) can be rewritten as

$$
\begin{aligned}
& p_{X}(1)^{M\left(\sigma_{1}\right)} p_{X}(0)^{N\left(\sigma_{1}\right)} \\
& \cdot\left(1-p_{X}(1)^{M\left(\sigma_{2}\right)-M\left(\sigma_{1}\right)} p_{X}(0)^{N\left(\sigma_{2}\right)-N\left(\sigma_{1}\right)}\right)=0,
\end{aligned}
$$

which holds if any of the three product terms on the left-hand side equal zero. It can be seen that $p_{X}(1)^{M\left(\sigma_{1}\right)}$ equals zero if and only if $p_{X}(1)=0$ and that $p_{X}(0)^{N\left(\sigma_{1}\right)}$ equals zero if and only if $p_{X}(1)=1$. Both values are not allowed for $p_{X}(1)$. Consequently, Equation (11) holds if and only if

$$
\begin{aligned}
1-p_{X}(1)^{M\left(\sigma_{2}\right)-M\left(\sigma_{1}\right)} p_{X}(0)^{N\left(\sigma_{2}\right)-N\left(\sigma_{1}\right)}=0 \\
\Longleftrightarrow p_{X}(1)^{M\left(\sigma_{2}\right)-M\left(\sigma_{1}\right)} p_{X}(0)^{N\left(\sigma_{2}\right)-N\left(\sigma_{1}\right)}=1
\end{aligned}
$$

holds. Since $p_{X}(1)<1$ and since $p_{X}(0)<1$, the product on the left-hand side of Equation (12) can only equal 1 for all $p_{X}(1)$ in the interval $(0,1)$ if $M\left(\sigma_{2}\right)=M\left(\sigma_{1}\right)$ and $N\left(\sigma_{2}\right)=N\left(\sigma_{1}\right)$. Consequently, $\mathrm{Cw}\left(\sigma_{1}\right)=\operatorname{Cw}\left(\sigma_{2}\right)$.

An algorithm that requires source word probabilities only (as, e.g., Huffman's algorithm) can be directly applied to a canonical source word multiset for a given $p_{X}(1)$. A similar concept is known for code word sets (or, in general, prefix-free codes), where only the code word lengths are relevant for the redundancy. Accordingly, the situation is more relaxed for code word sets than for source word sets, where the number of ones and zeros is relevant. The canonical Huffman codes $[4,10$, 20] take advantage of this property to efficiently represent and transmit Huffman codes. However, this concept works for arbitrary prefix-free codes. Since only the code word lengths are of interest, a canonical code word multiset shall be given as the multiset of code word lengths

$$
\operatorname{Cc}(C)=\{\ell(x) \mid x \in C\}_{b} .
$$

Moreover, it is well known that for any multiset of code word lengths, a prefix-free code can be constructed when the Kraft inequality [14] holds. A corresponding algorithm is given in Reference [5, Section 5.2]. Let

$$
\mathcal{V}_{i}^{c}=\operatorname{Cs}\left(\mathcal{P}_{i}\right) \times \Pi_{i} \times \operatorname{Cc}\left(\mathcal{P}_{i}\right)
$$

be the set of canonical $\mathrm{V} 2 \mathrm{~V}$ codes that have $i$ canonical source words and $i$ canonical code words. For a canonical code word multiset $C^{c}$, let $\operatorname{Rc}\left(C^{c}\right) \in \mathcal{P}$ be a code word set that is derived from $C^{c}$ so that $\operatorname{Cc}\left(\operatorname{Rc}\left(C^{c}\right)\right)=C^{c}$ holds. Analogously, for a canonical source word multiset $\mathcal{S}^{c}$, let $\operatorname{Rs}\left(\mathcal{S}^{c}\right) \in \mathcal{P}$ be a source word set that is derived from $\mathcal{S}^{c}$ so that $\operatorname{Cs}\left(\operatorname{Rs}\left(\mathcal{S}^{c}\right)\right)=\mathcal{S}^{c}$ holds. For a canonical V2V code $\left(\mathcal{S}^{c}, \pi^{\prime}, C^{c}\right) \in \mathcal{V}_{i}^{c}$, let $\operatorname{Rv}\left(\left(\mathcal{S}^{c}, \pi^{\prime}, C^{c}\right)\right) \in \mathcal{V}_{i}$ be a V2V code that is derived from $\left(\mathcal{S}^{c}, \pi^{\prime}, C^{c}\right)$ according to

$$
\operatorname{Rv}\left(\left(\mathcal{S}^{c}, \pi^{\prime}, C^{c}\right)\right)=\left(\operatorname{Rs}\left(\mathcal{S}^{c}\right), \pi^{\prime}, \operatorname{Rc}\left(C^{c}\right)\right)
$$

For a $\mathrm{V} 2 \mathrm{~V}$ code $v=(\mathcal{S}, \pi, C)$, let

$$
\operatorname{Cv}(v)=\left(\operatorname{Cs}(\mathcal{S}), \pi^{\prime}, \operatorname{Cc}(C)\right)
$$

be a canonical V2V code for which $\pi^{\prime}$ shall be chosen so that

$$
\bar{\ell}_{v}(X)=\bar{\ell}_{\mathrm{Rv}(\mathrm{Cv}(v))}(X)
$$


holds for $0<p_{X}(1)<1$. This shows that if for a V2V code $v$, the canonical $\mathrm{V} 2 \mathrm{~V}$ code $\mathrm{Cv}(v)$ is known, another $\mathrm{V} 2 \mathrm{~V}$ code $\mathrm{Rv}(\mathrm{Cv}(v))$ can be constructed from it that has the same average code length function as $v$. For a set of $\mathrm{V} 2 \mathrm{~V}$ codes $\mathcal{V}^{\prime}$, let

$$
\overline{\mathcal{L}}\left(\mathcal{V}^{\prime}\right)=\left\{\bar{\ell}_{v}(\cdot) \mid v \in \mathcal{V}^{\prime}\right\}
$$

be the set of average code length functions. Note that

$$
\overline{\mathcal{L}}\left(\mathcal{V}^{\prime}\right)=\overline{\mathcal{L}}\left(\operatorname{Rv}\left(\mathrm{Cv}\left(\mathcal{V}^{\prime}\right)\right)\right)
$$

holds, i.e., all redundancy functions of a set of $\mathrm{V} 2 \mathrm{~V}$ codes can also be derived from the corresponding set of canonical V2V codes.

\subsection{Prefix Merge Algorithm}

Generating a source word set from a canonical source word multiset, as required for function Rs $(\cdot)$, is a similar problem as generating a prefix-free code from code word lengths. However, it has the further constraint that the numbers of ones and zeros of the source words must be correct. While it is trivial to derive a canonical source word multiset from a source word set, the reverse way is not very intuitive. In the following, an algorithm is developed that allows the construction of a source word set $\mathcal{S}^{\prime}$ when only the canonical source word multiset $\operatorname{Cs}(\mathcal{S})$ of a source word set $\mathcal{S}$ is known. The algorithm ensures that $\operatorname{Cs}(\mathcal{S})=\operatorname{Cs}\left(\mathcal{S}^{\prime}\right)$ holds while $\mathcal{S}=\mathcal{S}^{\prime}$ does not need to hold. It is based on merging source words that only differ in the last bit (i.e., with identical prefixes) and is thus called prefix merge algorithm. Let $\mathcal{S}^{c}$ be a canonical source word multiset. From $\mathcal{S}^{c}$ derive the set

$$
\hat{\mathcal{S}}^{c}=\left\{(\hat{m}, \hat{n}) \in \mathcal{S}^{c} \mid \forall(m, n) \in \mathcal{S}^{c}: \hat{m}+\hat{n} \geq m+n\right\} .
$$

It is composed of all canonical source words of $\mathcal{S}^{c}$ that have maximum length. Furthermore, let

$$
\hat{\mathrm{X}}\left(\mathcal{S}^{c}\right)=\left(m^{\prime}, n^{\prime}\right) \in \hat{\mathcal{S}}^{c}
$$

such that

$$
\forall(m, n) \in \hat{\mathcal{S}}^{c}: m^{\prime} \geq m .
$$

In other words, $\hat{X}\left(\mathcal{S}^{c}\right)$ is a canonical source word with the maximum number of ones among the source words of maximum length of canonical source word multiset $\mathcal{S}^{c}$. Note that $\mathcal{S}^{c}$ may contain many instances of $\hat{\mathrm{X}}\left(\mathcal{S}^{c}\right)$. Let a further canonical source word be defined as $\hat{\mathrm{Y}}\left(\mathcal{S}^{c}\right)=\left(m^{\prime}-1, n^{\prime}+1\right)$.

The following property is similar to one of the requirement for optimum codes by Huffman as stated in Reference [11].

LEMmA 3.5. Let $\mathcal{S}$ be a source word set for which $\operatorname{Cs}(\mathcal{S})=\mathcal{S}^{c}$ holds and let $x$ be a source word in $\mathcal{S}$ for which $\mathrm{Cw}(x)=\hat{\mathrm{X}}\left(\mathcal{S}^{c}\right)$ holds. Then, $\mathcal{S}$ has a source word $y$ for which $\mathrm{Cw}(y)=\hat{\mathrm{Y}}\left(\mathcal{S}^{c}\right)$ holds and the leaf nodes associated with $x$ and $y$ in $\mathcal{S}$ are siblings.

Proof. Since a source tree is a full binary tree, each leaf node must have a sibling. Since the leaf node associated with source word $x$ is at the highest depth, it must have a sibling that is a leaf node. Since two source words, whose associated leaf nodes are siblings, differ only in the last bit, their number of ones must differ exactly by 1 . Since $x$ has the maximum number of ones among all source words of the same length, its sibling must have a 1 less and a zero more than $x$, which is the case for $y$.

When two leaf nodes of a source tree are siblings, they can be pruned, which means that they are removed and their common parent node becomes a new leaf node. Lemma 3.5 shows that when a source tree has a canonical source word multiset $\mathcal{S}^{c}$, then it also has two leaf nodes that are siblings and that have associated canonical source words $\hat{\mathrm{X}}\left(\mathcal{S}^{c}\right)$ and $\hat{\mathrm{Y}}\left(\mathcal{S}^{c}\right)$. Pruning of $\mathcal{S}^{c}$ corresponds to 


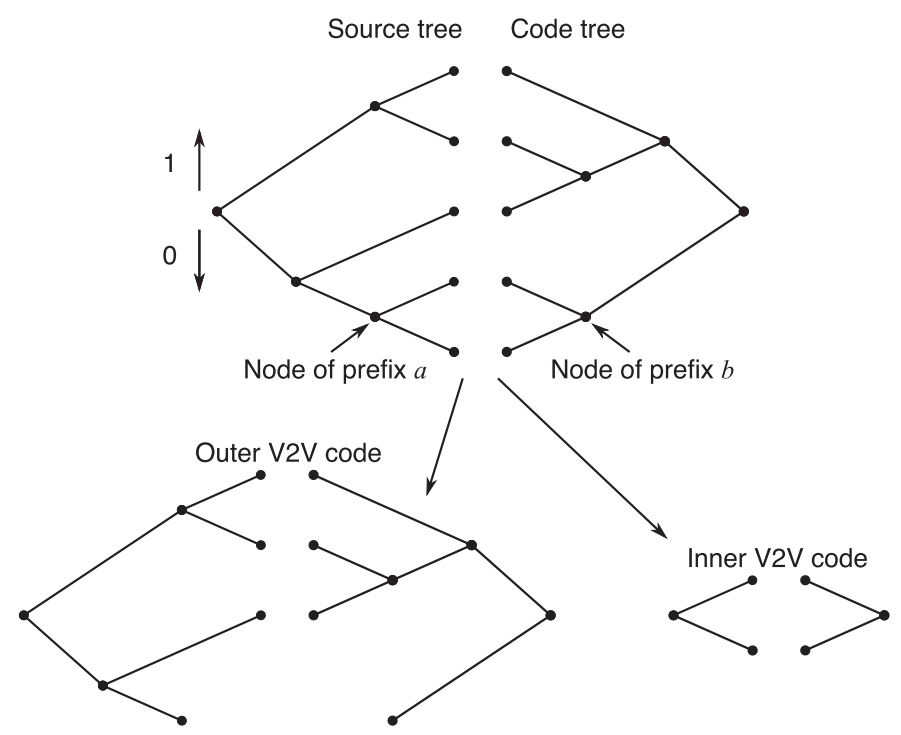

Fig. 1. Example for a composite V2V code.

replacing $\hat{X}\left(\mathcal{S}^{c}\right)$ and $\hat{\mathrm{Y}}\left(\mathcal{S}^{c}\right)$ with their common prefix, which shall be given as $\hat{Z}\left(\mathcal{S}^{c}\right)=\left(m^{\prime}-1, n^{\prime}\right)$. Let

$$
\Psi\left(\mathcal{S}^{c}\right)=\left(\mathcal{S}^{c} \backslash\left\{\hat{\mathrm{X}}\left(\mathcal{S}^{c}\right), \hat{\mathrm{Y}}\left(\mathcal{S}^{c}\right)\right\}\right) \cup\left\{\hat{\mathrm{Z}}\left(\mathcal{S}^{c}\right)\right\}
$$

be the canonical source word multiset $\mathcal{S}^{c}$ after one pruning step according to Lemma 3.5. A canonical source word multiset can repeatedly be pruned according to Equation (14) until only the root node is left. Obviously, a pruning step can be reversed to derive $\mathcal{S}^{c}$ from $\Psi\left(\mathcal{S}^{c}\right)$, which shall be called a growing step. The package merge algorithm recursively prunes a canonical source word multiset until only the root node is left. The resulting sequence of pruning steps is reversed and used to grow a source word set that corresponds to the desired canonical source word multiset. More precisely, consider a source word set $\mathcal{S}$ as it occurs during the growing procedure. Therefore, $\operatorname{Cs}(\mathcal{S})=\Psi\left(\mathcal{S}^{c}\right)$ shall hold. The growing step is applied to an arbitrary source word $\sigma \in \mathcal{S}$ with $\operatorname{Cw}(\sigma)=\hat{Z}\left(\mathcal{S}^{c}\right)$ to yield a source word set $\mathcal{S}^{\prime}$ for which $\operatorname{Cs}\left(\mathcal{S}^{\prime}\right)=\mathcal{S}^{c}$ holds. This procedure is carried out for each of the growing steps until the desired source tree is created.

\section{PRIME AND COMPOSITE V2V CODES}

In this section, the concept of prime and composite $\mathrm{V} 2 \mathrm{~V}$ codes is introduced.

Definition 4.1. A V2V code shall be composite if two prefixes $a$ and $b$ of nonzero length exist for which all of the following conditions are fulfilled:

- Two or more source words have prefix $a$

- Two or more code words have prefix $b$

- All source words with prefix $a$ are associated with code words with prefix $b$

- All code words with prefix $b$ are associated with source words with prefix $a$

A V2V code shall be prime if it is not composite.

A composite V2V code according to Definition 4.1 can be split into two V2V codes. An example is depicted in Figure 1. Prefix a corresponds to the internal node where the source tree is split, 
and prefix $b$ corresponds to the internal node where the code tree is split. The two separated subtrees constitute the so-called inner V2V code. In the remaining source and code tree, the two internal nodes where splitting was carried out are now leaf nodes. These are associated with each other and the resulting code shall be called outer V2V code. In the example of Figure 1, the outer and inner V2V codes are prime, since they cannot be split further. In principle, it is possible that inner and outer V2V codes are composite, and further splitting can be applied repeatedly until all resulting outer and inner $\mathrm{V} 2 \mathrm{~V}$ codes are prime. This is similar to the prime factors of a natural number. However, a V2V code is not uniquely described by its prime V2V codes, since it needs to be specified how the codes are connected. For example, connecting the inner V2V code of Figure 1 to a different pair of leaf nodes of the outer V2V code yields a new composite V2V code.

Encoding with a composite V2V code is identical to encoding with the outer V2V code and switching to the inner $\mathrm{V} 2 \mathrm{~V}$ code after a source word $a$ is encoded. After encoding a source word with the inner V2V code, it is switched back to the outer V2V code.

Consider the multiset of prime $\mathrm{V} 2 \mathrm{~V}$ codes that results from repeatedly splitting a V2V code. The question arises whether this multiset is unique for the $\mathrm{V} 2 \mathrm{~V}$ code. It turns out that this is the case, because all potential splitting points can be determined based on the original (unsplit) V2V code. Splitting at these points always results in the same subtrees regardless of the order in which the points are processed. Therefore, always the same multiset of prime V2V codes is obtained.

\subsection{Suboptimality of Composite V2V Codes}

Let a composite $\mathrm{V} 2 \mathrm{~V}$ code $v=(\mathcal{S}, \pi, C)$ be split into an inner and an outer $\mathrm{V} 2 \mathrm{~V}$ code according to Definition 4.1 (with prefixes $a$ and $b) . v_{I}=\left(\mathcal{S}_{I}, \pi_{I}, \mathcal{C}_{I}\right)$ shall be the the inner V2V code and $v_{O}=\left(\mathcal{S}_{O}, \pi_{O}, C_{O}\right)$ shall be the outer V2V code. Let $x \frown y$ be the concatenation of two binary words $x$ and $y$. Then, $\mathcal{S}$ can be written as

$$
\mathcal{S}=\left(\mathcal{S}_{O} \backslash\{a\}\right) \cup\left\{\hat{a} \widetilde{\sigma} \sigma \sigma \in \mathcal{S}_{I}\right\}
$$

and $C$ can be written as

$$
C=\left(C_{O} \backslash\{b\}\right) \cup\left\{b^{\frown} c \mid c \in C_{I}\right\} .
$$

Let $S, S_{I}, C_{I}, S_{O}$, and $C_{O}$ be the random variables associated with $\mathcal{S}, \mathcal{S}_{I}, C_{I}, \mathcal{S}_{O}$, and $C_{O}$, respectively, for encoding a binary i.i.d. source $\{X\}$. The average code length (4) of $v$ can be expressed as

$$
\begin{aligned}
\bar{\ell}_{v}(X)= & \frac{\bar{\ell}(C)}{\bar{\ell}(S)} \\
= & \frac{\bar{\ell}\left(C_{O}\right)-p_{S_{O}}(a) \ell(b)+\sum_{\sigma \in \mathcal{S}_{I}} p_{S}\left(a^{\frown} \sigma\right) \ell\left(k\left(a^{\frown} \sigma, v\right)\right)}{\bar{\ell}\left(S_{O}\right)-p_{S_{O}}(a) \ell(a)+\sum_{\sigma \in \mathcal{S}_{I}} p_{S}\left(a^{\frown} \sigma\right) \ell\left(a^{-} \sigma\right)} .
\end{aligned}
$$

For $\sigma \in \mathcal{S}_{I}$, observe that

$$
\begin{aligned}
p_{S}\left(a^{\frown} \sigma\right) & =p_{S_{O}}(a) p_{S_{I}}(\sigma), \\
\ell\left(k\left(a^{\frown} \sigma, v\right)\right) & =\ell(b)+\ell\left(k\left(\sigma, v_{I}\right)\right),
\end{aligned}
$$

and

$$
\sum_{\sigma \in \mathcal{S}_{I}} p_{S_{I}}(\sigma)=1
$$

hold. Thus, Equation (15) can be written as

$$
\bar{\ell}_{v}(X)=\frac{\bar{\ell}\left(C_{O}\right)+p_{S_{O}}(a) \bar{\ell}\left(C_{I}\right)}{\bar{\ell}\left(S_{O}\right)+p_{S_{O}}(a) \bar{\ell}\left(S_{I}\right)} .
$$


An intuitive interpretation can be given for Equation (16) as follows. $p_{S_{O}}(a) \bar{\ell}\left(S_{I}\right)$ corresponds to a lengthening of source word $a$, and $p_{S_{O}}(a) \bar{\ell}\left(C_{I}\right)$ corresponds to a lengthening of code word $b$.

LEMMA 4.2. Let $\bar{\ell}_{\min }(X)=\min \left\{\bar{\ell}_{v_{I}}(X), \bar{\ell}_{v_{O}}(X)\right\}$ and $\bar{\ell}_{\max }(X)=\max \left\{\bar{\ell}_{v_{I}}(X), \bar{\ell}_{v_{O}}(X)\right\}$. Then $\bar{\ell}_{\text {min }}(X) \leq \bar{\ell}_{v}(X) \leq \bar{\ell}_{\text {max }}(X)$. Furthermore, if $\bar{\ell}_{\text {min }}(X)<\bar{\ell}_{\text {max }}(X)$, then $\bar{\ell}_{\text {min }}(X)<\bar{\ell}_{v}(X)<$ $\bar{\ell}_{\text {max }}(X)$.

Proof. Let $a, b, c, d$ be real and positive. The mediant inequality states that if $a / b \leq c / d$, then

$$
\frac{a}{b} \leq \frac{a+c}{b+d} \leq \frac{c}{d}
$$

where $(a+c) /(b+d)$ is called mediant of $a / b$ and $c / d$. A proof can be found in the related literature (see Reference [9] for an overview). Since $\bar{\ell}\left(C_{O}\right), \bar{\ell}\left(S_{O}\right), \bar{\ell}\left(C_{I}\right), \bar{\ell}\left(S_{I}\right)$, and $p_{S_{O}}(a)$ are real and positive, $\bar{\ell}_{v}(X)$ is the mediant of the average code lengths of the inner and outer V2V code. Consequently,

holds if

$$
\bar{\ell}_{v_{O}}(X) \leq \bar{\ell}_{v}(X) \leq \bar{\ell}_{v_{I}}(X)
$$

$$
\bar{\ell}_{v_{O}}(X) \leq \bar{\ell}_{v_{I}}(X)
$$

and

holds if

$$
\bar{\ell}_{v_{O}}(X) \geq \bar{\ell}_{v}(X) \geq \bar{\ell}_{v_{I}}(X)
$$

$$
\bar{\ell}_{v_{O}}(X) \geq \bar{\ell}_{v_{I}}(X)
$$

which completes the proof of the first statement. The second statement can be proved in the same way (by excluding equality from all inequalities of the proof).

Lemma 4.2 states that each composite V2V code consists of at least one prime V2V code that has a lower or equal average code length as the composite V2V code. This property may be useful when searching a candidate set of $\mathrm{V} 2 \mathrm{~V}$ codes for minimum redundancy ${ }^{4}$ codes. Whenever a composite $\mathrm{V} 2 \mathrm{~V}$ code occurs and when it is guaranteed that the prime $\mathrm{V} 2 \mathrm{~V}$ codes it consists of are in the candidate set, the composite V2V code can be disregarded as minimum redundancy V2V code, because one of its prime codes has either lower or the same average code length as the composite V2V code.

\subsection{Primality Test for V2V Codes}

To benefit from the fact that composite V2V codes can be disregarded in particular V2V code design schemes, primality of the related codes needs to be tested. An algorithm for such a test can be derived from Definition 4.1 and is outlined in the following:

(1) Derive a set $A$ containing all prefixes of nonzero length that occur in source words of the source tree, not including the source words, themselves.

(2) For each prefix $B$ in set $A$, determine a subset $C$ that contains all source words that have this prefix.

(3) For the subset of source words $C$, derive a set $D$ that contains the code words that are associated with source words in $C$.

(4) Derive the common prefix $E$ of all code words in $D$.

(5) If $E$ is of nonzero length and if none of the other code words (not in $D$ ) also has prefix $E$, then the V2V code is composite.

\footnotetext{
${ }^{4}$ Note that codes with minimum redundancy are also codes with minimum average code length and vice versa according to Equation (8).
} 
In other words, it is iterated over all possible subtrees of the source tree, searching for a corresponding subtree in the code tree such that both subtrees form an inner V2V code according to Definition 4.1.

\subsection{Primality of Canonical V2V Codes}

The concept of prime and composite V2V codes can also be applied to canonical V2V codes. The following definition is proposed.

Definition 4.3. Consider a canonical V2V code $v^{c}$. If there exists at least one composite V2V code $v$ for which $\operatorname{Cv}(v)=v^{c}$ holds, then $v^{c}$ shall be considered composite. Otherwise, it shall be considered prime.

The motivation for defining primality of canonical V2V codes in this way is as follows. When one is interested in minimum redundancy codes, composite V2V codes can often be disregarded following the argument in Section 4.1. The existence of one composite V2V code $v$ is sufficient to prove that the associated canonical $\mathrm{V} 2 \mathrm{~V}$ code $v^{c}$ cannot have a lower redundancy than at least one of the $\mathrm{V} 2 \mathrm{~V}$ codes $v$ is composed of. This may be sufficient to disregard $v^{c}$ in a search for minimum redundancy codes. However, deciding whether a composite V2V code exists that corresponds to a given canonical V2V code seems to be a difficult task.

\subsection{Compositeness of Canonical Variable-to-Fixed Length Codes}

Consider the class of $\mathrm{V} 2 \mathrm{~V}$ codes where code trees are perfect binary trees, i.e., where all code words of a V2V code are of the same length. Such codes are known as variable-to-fixed length (V2F) codes. When canonical V2F codes are considered, it can be shown that they are always composite as follows.

Lemma 4.4. A canonical V2F code that has code words of length 2 or more is composite according to Definition 4.3 .

Proof. A composite V2F code can always be constructed from a canonical V2F code as follows when the code word are of length 2 or more. When all code words of a code tree are of the same length, it is irrelevant for the redundancy, how sources word are mapped to code words. Since each source tree must have at least two leaf nodes that are siblings, two sibling code words can be mapped to these sibling source words so that a composite V2F code results.

The same argumentation can be used to show that the so-called canonical fixed-to-variable length (F2V) codes, where all source words are of the same length, are always composite.

\section{DESIGN OF LIMITED SIZE V2V CODES WITH MINIMUM REDUNDANCY}

$\mathrm{V} 2 \mathrm{~V}$ codes can become arbitrarily large and, in principle, they can approach the source entropy arbitrarily close. In infinite subsets of $\mathcal{V}$, codes with minimal redundancy do not need to exist. An example is the set of F2V codes where all source words are of the same length. The infimum of the redundancies of all F2V codes is zero. A way to deal with infinite sets of V2V codes is to split them into finite subsets by introducing some parameter as, e.g., the source word length in the case of F2V codes. An algorithm for deriving minimum redundancy codes for these finite subsets is commonly referred to as optimal algorithm for the associated infinite subset. An example for this is the Tunstall algorithm [24], which finds optimal V2F codes where all code words are of the same length.

Practical applications usually introduce restrictions to the properties of V2V codes. These could be of various kinds as a limited source or code tree height, a limited number of leaf nodes for 
source and code tree, a minimum number of source symbols per code word, and so forth. Given such constraints, the question arises, how minimum redundancy codes can be found? In the related literature, several algorithms for V2V code design evolved [6-8, 21-23]. However, algorithms for finding minimum redundancy codes are all based on exhaustive search. A comprehensive survey can be found in Reference [1]. In this section, Huffman's algorithm, the package merge algorithm, and the concept of canonical V2V codes are employed to greatly reduce the complexity of the exhaustive search.

\subsection{Minimum Redundancy Canonical V2V Codes}

$\mathrm{V} 2 \mathrm{~V}$ codes of limited size (in some sense), can be described by a finite subset of $\mathcal{V}$ that contains all $\mathrm{V} 2 \mathrm{~V}$ codes that meet the desired size criteria. Let $\mathcal{V}^{\prime}$ be such a subset. The set of all minimum redundancy $\mathrm{V} 2 \mathrm{~V}$ codes for a particular $p_{X}(1)$ are then given as

$$
\mathrm{V}_{\text {min }}\left(\mathcal{V}^{\prime}, X\right)=\left\{v \in \mathcal{V}^{\prime} \mid \forall v^{\prime} \in \mathcal{V}^{\prime}: \bar{\ell}_{v}(X) \leq \bar{\ell}_{v^{\prime}}(X)\right\}
$$

It may be sufficient to only consider the minimum redundancy canonical $\mathrm{V} 2 \mathrm{~V}$ codes $\mathrm{Cv}\left(\mathrm{V}_{\text {min }}\right.$ $\left.\left(\mathcal{V}^{\prime}, X\right)\right)$, because, according to Equation (13), they have the same average code length functions as $\mathrm{V} 2 \mathrm{~V}$ codes in $\mathrm{V}_{\min }\left(\mathcal{V}^{\prime}, X\right)$. Analogously, $\overline{\mathcal{L}}\left(\operatorname{Rv}\left(\mathrm{Cv}\left(\mathcal{V}^{\prime}\right)\right)\right)$ contains the same average code length functions as $\overline{\mathcal{L}}\left(\mathcal{V}^{\prime}\right)$, and therefore

$$
\mathrm{Cv}\left(\mathrm{V}_{\text {min }}\left(\mathcal{V}^{\prime}, X\right)\right)=\mathrm{Cv}\left(\mathrm{V}_{\text {min }}\left(\operatorname{Rv}\left(\mathrm{Cv}\left(\mathcal{V}^{\prime}\right)\right), X\right)\right) .
$$

Solving Reference (17) may be costly when $\mathcal{V}^{\prime}$ contains a large number of V2V codes. Whenever $\operatorname{Rv}\left(\mathrm{Cv}\left(\mathcal{V}^{\prime}\right)\right)$ contains substantially fewer $\mathrm{V} 2 \mathrm{~V}$ codes than $\mathcal{V}^{\prime}$, solving Reference (18) is substantially less costly than solving Reference (17). In this way, canonical V2V codes can reduce the complexity of an exhaustive search for minimum redundancy V2V codes.

\subsection{V2V Codes with Huffman or Package Merge Code Trees}

Huffman's algorithm [11] as well as the more general package merge algorithm [15] may be beneficial in finding $\mathrm{V}_{\min }\left(\mathcal{V}^{\prime}, X\right)$ when $\mathcal{V}^{\prime}$ has particular properties. For a $\mathrm{V} 2 \mathrm{~V}$ code $v=(\mathcal{S}, \pi, C)$, let

$$
\operatorname{St}(v)=\mathcal{S}
$$

yield the source word set. Let $\mathrm{Hu}(\mathcal{S}, X)$ yield a V2V code with a code word set, derived by Huffman's algorithm, for a given source word set $\mathcal{S}$ and for a given $p_{X}(1)$. Analogously, let $\operatorname{Pm}(\mathcal{S}, X, d)$ be a V2V code with a code word set derived by the package merge algorithm with maximum code tree height $d$ for a given source word set $\mathcal{S}$ and for a given $p_{X}(1)$. The $\operatorname{set} \operatorname{Hu}\left(\operatorname{St}\left(\mathcal{V}^{\prime}\right), X\right)$ contains all V2V codes constructed by combining each source word set in V2V codes of $\mathcal{V}^{\prime}$ with the corresponding Huffman code word sets for a given $p_{X}(1)$. For a given source word set, Huffman's algorithm produces a minimum redundancy code word set. Consequently, if

$$
\mathrm{Hu}\left(\operatorname{St}\left(\mathcal{V}^{\prime}\right), X\right) \subseteq \mathcal{V}^{\prime}
$$

holds, then

$$
\overline{\mathcal{L}}\left(\mathrm{V}_{\text {min }}\left(\mathcal{V}^{\prime}, X\right)\right)=\overline{\mathcal{L}}\left(\mathrm{V}_{\text {min }}\left(\mathrm{Hu}\left(\mathrm{St}\left(\mathcal{V}^{\prime}\right), X\right), X\right)\right)
$$

holds as well and $\mathrm{V}_{\min }\left(\mathrm{Hu}\left(\mathrm{St}\left(\mathcal{V}^{\prime}\right), X\right), X\right)$ contains at least one minimum redundancy code of $\mathcal{V}^{\prime}$ for a given $p_{X}(1)$. Equation (19) can be extended by the concept of canonical source trees. If

$$
\operatorname{Hu}\left(\operatorname{Rs}\left(\operatorname{Cs}\left(\operatorname{St}\left(\mathcal{V}^{\prime}\right)\right)\right), X\right) \subseteq \mathcal{V}^{\prime}
$$

holds, then

$$
\overline{\mathcal{L}}\left(\mathrm{V}_{\text {min }}\left(\mathcal{V}^{\prime}, X\right)\right)=\overline{\mathcal{L}}\left(\mathrm{V}_{\text {min }}\left(\mathrm{Hu}\left(\operatorname{Rs}\left(\operatorname{Cs}\left(\operatorname{St}\left(\mathcal{V}^{\prime}\right)\right)\right), X\right), X\right)\right)
$$


holds as well, and $\mathrm{V}_{\text {min }}\left(\mathrm{Hu}\left(\operatorname{Rs}\left(\operatorname{Cs}\left(\operatorname{St}\left(\mathcal{V}^{\prime}\right)\right)\right), X\right), X\right)$ contains at least one minimum redundancy $\mathrm{V} 2 \mathrm{~V}$ code of $\mathcal{V}^{\prime}$ for a given $p_{X}(1)$.

\section{SELECTED SETS OF SIZE-LIMITED V2V CODES}

In the following, various types of size limitations that can apply to $\mathrm{V} 2 \mathrm{~V}$ codes are discussed, and corresponding subsets of $\mathcal{V}$ are defined. The cardinalities of these subsets are derived as they are the most relevant factor for the computational complexity of finding minimum redundancy codes. Whenever applicable, the concept canonical source trees and Huffman or package merge code trees is employed to reduce this computational complexity.

\subsection{Leaf-limited V2V Codes}

The set of all V2V codes with the number of leaf nodes $|\mathcal{S}|$ (of source tree or code tree) not exceeding a predefined maximum count $x$ is given as

$$
\tilde{V}_{x}=\mathcal{V}_{2} \cup \mathcal{V}_{3} \cup \cdots \cup \mathcal{V}_{x}
$$

and it shall be called set of leaf-limited variable-to-variable length (LV2V) codes. Since code trees in $\tilde{V}_{x}$ are only restricted by the number of leaf nodes, minimum redundancy LV2V codes have Huffman code trees. Consequently, Equation (19) holds for $\tilde{V}_{x}$. Note that the set of all source word sets in $\tilde{V}_{x}$ are given as

$$
\tilde{\mathcal{P}}_{x}=\operatorname{St}\left(\tilde{\mathcal{V}}_{x}\right)=\mathcal{P}_{2} \cup \mathcal{P}_{3} \cup \cdots \cup \mathcal{P}_{x} .
$$

Since all possible V2V codes with up to $x$ leaf nodes in source and code tree are contained in $\tilde{V}_{x}$, all possible V2V codes with source trees $\operatorname{Rs}\left(\operatorname{Cs}\left(\tilde{\mathcal{P}}_{x}\right)\right)$ are also contained in $\tilde{V}_{x}$ and thus Equation (20) holds as well for $\tilde{V}_{x}$.

To the authors' best knowledge, an efficient algorithm for finding LV2V codes with minimum redundancy is unknown. However, since Equation (20) holds for $\tilde{V}_{x}$,

$$
\mathrm{V}_{\text {min }}\left(\operatorname{Hu}\left(\operatorname{Rs}\left(\operatorname{Cs}\left(\tilde{\mathcal{P}}_{x}\right)\right), X\right), X\right)
$$

contains at least one minimum redundancy LV2V code for a given $p_{X}(1)$. The cardinality of $\operatorname{Cs}\left(\tilde{\mathcal{P}}_{x}\right)$ is therefore most relevant for the computational complexity of finding Equation (21).

Construction rules for the set $\mathcal{P}_{x}$ can be found in the related literature as, e.g., in Reference [18, p. 127] where it is also shown that

$$
\left|\mathcal{P}_{x}\right|=\zeta_{x-1}
$$

with

$$
\zeta_{n}=\frac{(2 n) !}{n !(n+1) !}
$$

being the Catalan numbers (see Reference [18, p. 114]). Thus,

$$
\left|\tilde{\mathcal{P}}_{x}\right|=\sum_{n=1}^{x-1} \zeta_{n}
$$

is an indicator for the computational complexity of deriving $\mathrm{V}_{\min }\left(\mathrm{Hu}\left(\tilde{\mathcal{P}}_{x}, X\right), X\right)$. When also the concept of canonical source trees is employed according to Equation (21), the computational complexity can further be decreased since

$$
\left|\operatorname{Cs}\left(\tilde{\mathcal{P}}_{x}\right)\right| \leq\left|\tilde{\mathcal{P}}_{x}\right| .
$$

Unfortunately, no simple closed-form expression for deriving $\left|\operatorname{Cs}\left(\tilde{\mathcal{P}}_{x}\right)\right|$ could be found. 


\subsection{Source and/or Code-height-limited V2V Codes}

The next subsets of $\mathcal{V}$ to be discussed limit the maximum source and/or code tree height. Let

$$
\hat{\mathcal{V}}_{x}=\{(\mathcal{S}, \pi, C) \in \mathcal{V} \mid \forall s \in \mathcal{S}: \ell(s) \leq x\}
$$

be the set of all $\mathrm{V} 2 \mathrm{~V}$ codes with limited maximum source tree height $x$. They shall be called sourceheight-limited variable-to-variable length (SV2V) codes. Analogously, the set of V2V codes with the code word length limited to $x$ is given as

$$
\overline{\mathcal{V}}_{x}=\{(\mathcal{S}, \pi, C) \in \mathcal{V} \mid \forall c \in C: \ell(c) \leq x\},
$$

and these are called code-height-limited variable-to-variable length $(\mathrm{CV} 2 \mathrm{~V})$ codes. Correspondingly, V2V codes with limited source and code tree height (SCV2V codes) are given as

$$
\hat{\bar{V}}_{x}^{y}=\hat{V}_{x} \cap \bar{V}_{y}
$$

where the maximum source tree height is $x$ and the maximum code tree height is $y$. In the following, design strategies for SV2V codes, CV2V codes, and SCV2V codes are discussed. Huffman's algorithm is employed to find code trees for SV2V codes and the package merge algorithm finds height-limited code trees for $\mathrm{CV} 2 \mathrm{~V}$ codes and SCV2V codes. The difficulty lies in the set of source trees that needs to be considered, which is discussed in the following.

6.2.1 SV2V Codes. The set of all source word sets that occur in $\hat{V}_{x}$ are given as

$$
\hat{\mathcal{P}}_{x}=\operatorname{St}\left(\hat{\mathcal{V}}_{x}\right)=\{y \in \mathcal{P} \mid \forall z \in y: \ell(z) \leq x\} .
$$

Since $\hat{\mathcal{V}}_{x}$ contains all possible V2V codes with source trees of height up to $x$, it must also contain all possible V2V codes with source trees $\operatorname{Rs}\left(\operatorname{Cs}\left(\hat{\mathcal{P}}_{x}\right)\right)$. Consequently, Equations (19) and (20) hold for $\hat{V}_{x}$, and

$$
\mathrm{V}_{\text {min }}\left(\mathrm{Hu}\left(\operatorname{Rs}\left(\operatorname{Cs}\left(\hat{\mathcal{P}}_{x}\right)\right), X\right), X\right)
$$

contains at least one minimum redundancy SV2V code for a given $p_{X}(1)$. The cardinality of $\operatorname{Cs}\left(\hat{\mathcal{P}}_{x}\right)$ is most relevant for the computational complexity of finding Equation (23).

As shown in Reference [3, p. 717], $\left|\hat{\mathcal{P}}_{x}\right|$ can be calculated from $\left|\hat{\mathcal{P}}_{x-1}\right|$ according to

$$
\left|\hat{\mathcal{P}}_{x}\right|=\left(1+\left|\hat{\mathcal{P}}_{x-1}\right|\right)^{2},
$$

where the number of word sets $\left|\hat{\mathcal{P}}_{1}\right|$ of height 1 equals 1 . A lower bound on $\left|\hat{\mathcal{P}}_{x}\right|$ can be defined using the approximation

$$
\left|\hat{\mathcal{P}}_{x}\right|=\left(1+\left|\hat{\mathcal{P}}_{x-1}\right|\right)^{2}>\left|\hat{\mathcal{P}}_{x-1}\right|^{2} .
$$

Starting with $\left|\hat{\mathcal{P}}_{2}\right|=4$, the lower bound is given as

$$
\left|\hat{\mathcal{P}}_{x}\right|>2^{2^{x-1}}
$$

for $x>2$. Equation (24) shows that $\left|\hat{\mathcal{P}}_{x}\right|$ has at least double-exponential growth $O\left(2^{2^{n}}\right)$, which makes finding

$$
\mathrm{V}_{\text {min }}\left(\mathrm{Hu}\left(\hat{\mathcal{P}}_{x}, X\right), X\right)
$$

practically infeasible for $x>6$ (since $\left.\left|\hat{\mathcal{P}}_{7}\right|>4.4 \cdot 10^{22}\right)$. As for LV2V codes, no closed-form expression for deriving $\left|\operatorname{Cs}\left(\hat{\mathcal{P}}_{x}\right)\right|$ could be found, but these values can be derived by simulation. For $2 \leq x \leq 6$, these values are listed in Table 1. Comparing $\left|\hat{\mathcal{P}}_{x}\right|$ with the corresponding $\left|\operatorname{Cs}\left(\hat{\mathcal{P}}_{x}\right)\right|$ reveals that the concept of canonical source word multisets can greatly reduce the complexity of finding minimum redundancy SV2V codes. For example, $\left|\hat{\mathcal{P}}_{6}\right|$ is approximately 47,196 times as large as $\left|\operatorname{Cs}\left(\hat{\mathcal{P}}_{6}\right)\right|$. Employing this concept made it possible to find many of the minimum redundancy $\mathrm{V} 2 \mathrm{~V}$ codes that are presented in Section 7.1. 
Table 1. Comparison of Counts of Full Binary Trees $\left|\hat{\mathcal{P}}_{x}\right|$ and Associated Canonical Source Word Multisets $\left|\operatorname{Cs}\left(\hat{\mathcal{P}}_{x}\right)\right|$ for $2 \leq x \leq 6$

\begin{tabular}{|l|c|c|c|c|c|}
\hline$x$ & 2 & 3 & 4 & 5 & 6 \\
\hline$\left|\hat{\mathcal{P}}_{x}\right|$ & 4 & 25 & 676 & 458,329 & $210,066,388,900$ \\
\hline$\left|\operatorname{Cs}\left(\hat{\mathcal{P}}_{x}\right)\right|$ & 4 & 21 & 253 & 12,360 & $4,450,860$ \\
\hline$\frac{\left|\hat{\mathcal{P}}_{i}\right|}{\left|\operatorname{Cs}\left(\hat{\mathcal{P}}_{i}\right)\right|}$ & 1 & $\approx 1.2$ & $\approx 2.7$ & $\approx 37.1$ & $\approx 47,196.8$ \\
\hline
\end{tabular}

6.2.2 CV2V Codes. For a given $p_{X}(1)$, minimum redundancy CV2V codes have code word sets that can be derived by the package merge algorithm. The source tree height is only limited by the number of code words, and the number of code words is only limited by the maximum code tree height. More precisely, for a maximum code tree height $x$, the maximum possible number of leaf nodes (of source or code tree) is $2^{x}$. Obviously, when a code tree of height $x$ has $2^{x}$ leaf nodes, all of them must be at the same level, which leads to a V2F code. For $x>1$, a canonical V2F code is always composite according to Lemma 4.4. According to Lemma 4.2, one of the prime V2V codes it consists of has the same or a lower average code length, so that it can be disregarded as minimum redundancy $\mathrm{CV} 2 \mathrm{~V}$ code. Consequently, at least one minimum redundancy $\mathrm{CV} 2 \mathrm{~V}$ code is contained in

$$
\mathrm{V}_{\min }\left(\operatorname{Pm}\left(\tilde{\mathcal{P}}_{2^{x}-1}, X, x\right), X\right)
$$

for a given $p_{X}(1)$. The cardinalities $\left|\tilde{\mathscr{P}}_{2^{x}-1}\right|$ grow much faster in $x$ than source word sets for SV2V codes. Already CV2V codes with $x=5$ can hardly be derived by exhaustive search, since $\left|\tilde{\mathcal{P}}_{31}\right|>5.1 \cdot 10^{15}$. Obviously, it is sufficient to only consider canonical source word multisets $\operatorname{Cs}\left(\tilde{\mathcal{P}}_{2^{x}-1}\right)$ for finding minimum redundancy CV2V codes. An exhaustive search over $\operatorname{Cs}\left(\tilde{\mathcal{P}}_{31}\right)$ may be feasible, but this remains to be shown.

6.2.3 SCV2V Codes. According to Equation (22), SCV2V codes are a subset of CV2V codes and SV2V codes. Height-limited code trees are combined with height-limited source trees. Consequently, source word sets of V2V codes in $\hat{\bar{V}}_{x}^{y}$ are $\hat{\mathcal{P}}_{x} \cap \tilde{\mathcal{P}}_{2^{y}}$. They have a limited source word count and a limited source word length. Following the same argumentation as for CV2V codes, source word sets with $2^{y}$ leaf nodes can be neglected, since they can only be mapped to a perfect binary code tree, which yields a V2F code. Hence, the source trees of interest are $\hat{\mathcal{P}}_{x} \cap \tilde{\mathcal{P}}_{2 y-1}$. Furthermore, only canonical source word multisets need to be considered, and, therefore, at least one minimum redundancy $\mathrm{V} 2 \mathrm{~V}$ code of $\hat{\bar{V}}_{x}^{y}$ is contained in

$$
\mathrm{V}_{\text {min }}\left(\operatorname{Pm}\left(\operatorname{Rs}\left(\operatorname{Cs}\left(\hat{\mathcal{P}}_{x} \cap \tilde{\mathcal{P}}_{2^{y}-1}\right)\right), X, y\right), X\right)
$$

for a given $p_{X}(1)$. Cardinalities of $\operatorname{Cs}\left(\hat{\mathcal{P}}_{x} \cap \tilde{\mathcal{P}}_{2^{y}-1}\right)$ are most relevant for the computational complexity of finding Reference (25). For $3 \leq x, y \leq 6$, these cardinalities were derived by simulation and are listed in Table 2.

Limiting source and code word length of a V2V code is of particular interest for an implementation of a V2V encoder or decoder that is based on a lookup table. For example, a V2V encoder may use $k$ input symbols to access a table with $2^{k}$ elements. The addressed element stores the corresponding code word and the length of the source word. Then it removes the source word from the input sequence and outputs the code word. Using this approach, a table with $2^{k}$ elements is able to implement all V2V codes with maximum source tree height of $k$. Consequently, minimum redundancy $\mathrm{V} 2 \mathrm{~V}$ codes for such a lookup table have a limited source tree height. The same concept 
Table 2. Cardinalities of $\operatorname{Cs}\left(\hat{\mathcal{P}}_{x} \cap \tilde{\mathcal{P}}_{2^{y}-1}\right)$

\begin{tabular}{|c|r|r|r|r|r|}
\cline { 3 - 6 } \multicolumn{2}{c|}{} & \multicolumn{4}{c|}{$y$} \\
\cline { 3 - 7 } \multicolumn{2}{c|}{} & 3 & 4 & 5 & 6 \\
\hline \multirow{4}{*}{$x$} & 3 & 20 & 21 & 21 & 21 \\
\cline { 2 - 6 } & 4 & 67 & 252 & 253 & 253 \\
\cline { 2 - 6 } & 5 & 127 & 4,369 & 12,359 & 12,360 \\
\cline { 2 - 6 } & 6 & 159 & 31,865 & $2,167,044$ & $4,450,859$ \\
\hline
\end{tabular}

can be used for a decoder implementation when source and code tree are exchanged. Then, minimum redundancy V2V codes for such a lookup table have a limited code tree height.

\section{REDUNDANCY OF SIZE-LIMITED V2V CODES}

In the previous section, various types of $\mathrm{V} 2 \mathrm{~V}$ codes were discussed, and strategies for finding minimum redundancy $\mathrm{V} 2 \mathrm{~V}$ codes of these types were proposed. The approaches were all based on predefining a value for $p_{X}(1)$ and executing one of the algorithms for this value. It may, however, be of interest to find minimum redundancy codes for all $0<p_{X}(1) \leq 0.5$. For example, the PIPE coding concept $[16,17,25]$ would require a set of $\mathrm{V} 2 \mathrm{~V}$ codes that jointly cover the probability interval $(0,0.5]$. An intuitive approach is to select an appropriate set of distinct values for $p_{X}(1)$ and execute the algorithm for each of the values. However, it can happen that some V2V codes that have minimum redundancy for some values of $p_{X}(1)$ are not discovered in this way. This may be irrelevant for a practical application, but, from a theoretical point of view, it may be interesting to find the set of all $\mathrm{V} 2 \mathrm{~V}$ codes that have minimum redundancy for at least one value of $p_{X}(1)$. For a candidate set of $\mathrm{V} 2 \mathrm{~V}$ codes $\mathcal{V}^{\prime}$, these $\mathrm{V} 2 \mathrm{~V}$ codes are given as subset

$$
\begin{aligned}
\mathcal{M}\left(\mathcal{V}^{\prime}\right)= & \left\{v \in \mathcal{V}^{\prime} \mid \exists p_{X}(1):\right. \\
& \left.0<p_{X}(1) \leq 0.5 \wedge\left(\forall u \in \mathcal{V}^{\prime}: \bar{\ell}_{v}(X) \leq \bar{\ell}_{u}(X)\right)\right\} .
\end{aligned}
$$

Deriving $\mathcal{M}\left(\mathcal{V}^{\prime}\right)$ can be based on comparing average code length functions, which shall be outlined in the following. Consider two V2V codes $v_{1}=\left(\mathcal{S}_{1}, \pi_{1}, C_{1}\right)$ and $v_{2}=\left(\mathcal{S}_{2}, \pi_{2}, C_{2}\right)$. They have the same average code length when

$$
\bar{\ell}_{v_{1}}(X)=\bar{\ell}_{v_{2}}(X)
$$

holds. Let $S_{1}, C_{1}, S_{2}$, and $C_{2}$ be the random variables associated with $\mathcal{S}_{1}, C_{1}, \mathcal{S}_{2}$, and $C_{2}$, respectively, for encoding a binary i.i.d. source $\{X\}$. Then, Equation (26) can be written as

$$
\begin{aligned}
& \frac{\bar{\ell}\left(C_{1}\right)}{\bar{\ell}\left(S_{1}\right)}=\frac{\bar{\ell}\left(C_{2}\right)}{\bar{\ell}\left(S_{2}\right)} \\
\Longleftrightarrow & \bar{\ell}\left(C_{1}\right) \bar{\ell}\left(S_{2}\right)-\bar{\ell}\left(C_{2}\right) \bar{\ell}\left(S_{1}\right)=0,
\end{aligned}
$$

with

$$
\operatorname{Poly}\left(v_{1}, v_{2}\right)=\bar{\ell}\left(C_{1}\right) \bar{\ell}\left(S_{2}\right)-\bar{\ell}\left(C_{2}\right) \bar{\ell}\left(S_{1}\right),
$$

and Equation (27) becomes

$$
\operatorname{Poly}\left(v_{1}, v_{2}\right)=0
$$

The left-hand side of Equation (28) is a polynomial in $p_{X}(1)$ and Equation (28) holds for the roots of the polynomial. Between two consecutive real polynomial roots, the polynomial is either positive or negative, which means that one of the two V2V codes $v_{1}$ or $v_{2}$ has a lower average code length for all values between the two roots than the other V2V code. Consequently, each two consecutive real polynomial roots can be interpreted as boundaries of an interval in which one of the two 


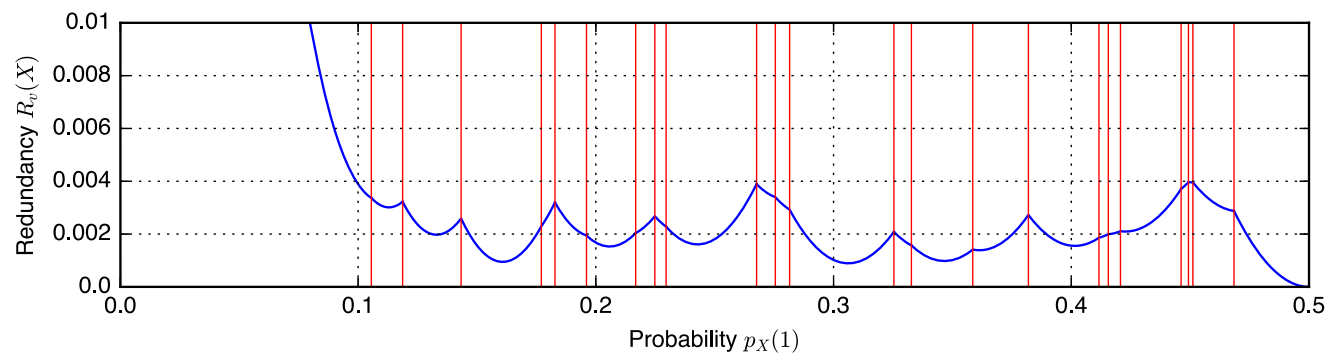

Fig. 2. Redundancy of minimum redundancy SCV2V codes with source and code tree height limited to 6.

V2V codes has the lower average code length. Moreover, it can also happen that a V2V code has minimum redundancy at a particular polynomial root only. Result of this procedure is a subdivision of the probability interval $(0,0.5]$ into subintervals so that each subinterval is associated with the $\mathrm{V} 2 \mathrm{~V}$ code with the lower redundancy. Without going into detail, the procedure can be repeated for each of the subintervals with further V2V codes to find minimum redundancy V2V codes among all tested ones. A detailed description of the concept can be found in Reference [13].

Unfortunately, most of the search strategies for $\mathrm{V} 2 \mathrm{~V}$ codes of the previous section require us to employ, e.g., Huffman's algorithm or the package merge algorithm, so that the above procedure cannot be employed. However, the idea of working with polynomials instead of working with fixed probability values can be extended to arbitrary algorithms, which leads to the concept of polynomial-based algorithms. This concept is proposed in Reference [13], where it is employed for jointly deriving V2V codes and associated probability intervals. The experimental results presented in this section are derived by employing this concept.

\subsection{Redundancy Comparison of Size-limited V2V Codes}

In the following, minimum redundancy $\mathrm{V} 2 \mathrm{~V}$ codes of limited size and associated probability intervals are evaluated by employing the concept of polynomial-based algorithms as described in Reference [13]. At first, SCV2V codes with source and code tree height limited to 6 shall be evaluated. Executing a polynomial-based version of the package merge algorithm over the set of 4,450,859 candidate canonical source trees, as derived in Section 6, leads to 24 minimum redundancy codes with distinct average code length functions. Interestingly, each of the found V2V codes has minimum redundancy for exactly one subinterval. It is unclear whether this must necessarily be the case or whether a V2V code can also have minimum redundancy in several probability intervals that are not contiguous. Figure 2 shows their redundancies and associated probability intervals as vertical lines. Note that the redundancy of a code is only depicted in its associated interval. The corresponding canonical SCV2V codes are listed in Reference [13]. It can be seen that the redundancy for $p_{X}(1)>0.1$ ranges from approximately 0.001 to 0.004 bit per binary input symbol from $H(X)$, which is remarkably low.

Next, several types of $\mathrm{V} 2 \mathrm{~V}$ codes shall be evaluated for a V2V encoder implementation that is based on a lookup table with $2^{n}$ rows so that source words may be up to $n$ bits long. The required memory for the lookup table grows exponentially with the source word length and linearly with the code word length. Therefore, the code word length is less critical and shall not be restricted. The minimum achievable redundancy for a lookup-table-based encoder with $n=5$ shall be derived for SV2V codes, LV2V codes, and so-called F2V codes. Note that F2V codes have a constant source word length and a Huffman code tree. Since the lookup table can handle a maximum source word length of 5, it can implement all LV2V codes with up to six leaf nodes in the source or code tree. 


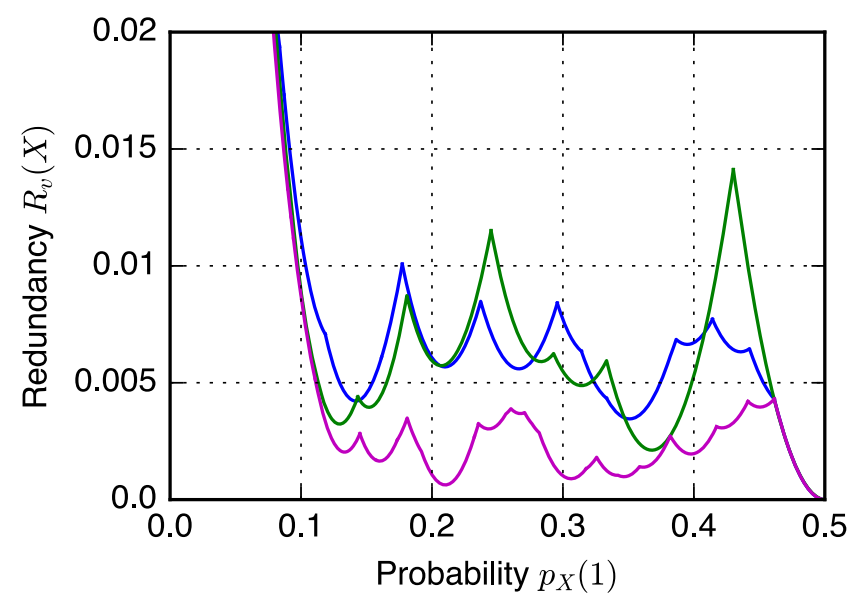

Fig. 3. Redundancy comparison of minimum redundancy $V 2 \mathrm{~V}$ codes, blue, F2V codes with a source word length up to 5; green, LV2V codes with up to six leaf nodes in source or code tree; magenta, SV2V codes with a source tree height of up to 5 .

Obviously, SV2V codes and F2V codes with a source word length of up to 5 can also be implemented. Figure 3 compares minimum redundancy codes of these three classes. For a better readability, probability interval boundaries are not depicted but can be looked up in Reference [13], as well as all related canonical V2V codes. While LV2V codes and F2V codes have a comparable redundancy, SV2V codes have a considerably lower redundancy. Obviously, SV2V codes must have the lowest redundancy, because any V2V code that can be implemented with a lookup table with $2^{n}$ rows is also an SV2V code where the source word length does not exceed $n$. Conversely, each SV2V code with the source word length not exceeding $n$ can be implemented with a lookup table with $2^{n}$ rows. Therefore, SV2V codes can best exploit the described lookup table-based encoder.

\section{NONZERO REDUNDANCY OF FINITE SIZE V2V CODES}

It can be shown that Equation (9) is always greater zero for $0<p_{X}(1)<0.5$. Stubley shows in Reference [21, Section 7.1.3] that there exist particular values of $p_{X}(1)$ for which the redundancy of a V2V code cannot be zero, and in the following, it is proven by contradiction that the redundancy of a V2V code is always nonzero for $0<p_{X}(1)<0.5$ and $p_{X}(1) \in \mathbb{R}$. Obviously, $R_{v}(X)=0$ if and only if $D(S \| \hat{S})=0$ by Equation (9).

LEMMA 8.1. Let $\mathcal{S}=\left\{\sigma_{1}, \sigma_{2}, \ldots, \sigma_{n}\right\}$ be the set of source words of a source tree. There exists exactly one source word $\sigma_{\alpha}$ that only consists of ones and one source word $\sigma_{\beta}$ that only consists of zeros.

Proof. Since a source tree is a full binary tree, each node has 0 or 2 child nodes. Consequently, it is possible to traverse the tree from root by always selecting 1 as next symbol, yielding $\sigma_{\alpha}$, or by always selecting 0 as next symbol, yielding $\sigma_{\beta}$.

Assume that there exists a redundancy-free V2V code with $0<p_{X}(1)<0.5$. Let $\sigma_{\alpha}$ and $\sigma_{\beta}$ be as in Lemma 8.1 with source word lengths $a=\ell\left(\sigma_{\alpha}\right)$ and $b=\ell\left(\sigma_{\beta}\right)$. Substituting the probabilities of source words (1) for $\sigma_{\alpha}$ and $\sigma_{\beta}$ into Equation (7) yields

$$
\begin{array}{llr}
2^{-c}=p_{X}(1)^{a} & \Longleftrightarrow & 2^{-\frac{c}{a}}=p_{X}(1), \\
2^{-d}=\left(1-p_{X}(1)\right)^{b} & \Longleftrightarrow & 1-2^{-\frac{d}{b}}=p_{X}(1),
\end{array}
$$


where $c$ and $d$ are the lengths of the code words to be associated with $\sigma_{\alpha}$ and $\sigma_{\beta}$, respectively. The following Lemma is taken from Reference [2].

Lemma 8.2. Let $a_{1}, a_{2}, \ldots, a_{n}, b_{1}, b_{2}, \ldots, b_{n} \in \mathbb{Q}^{+}$, and $k \in \mathbb{Z}^{+}$. A sum $\sum_{i=1}^{n} a_{i} \sqrt[k]{b_{i}}=M \in \mathbb{Z}$ cannot occur if at least one $\sqrt[k]{b_{i}}$ is irrational.

Proof. See the section titled "Higher powers" in Reference [2].

THEOREM 8.3. Let $\sigma_{\alpha}$ and $\sigma_{\beta}$ be source words according to Lemma 8.1 with length $a=\ell\left(\sigma_{\alpha}\right)>0$ and $b=\ell\left(\sigma_{\beta}\right)>0$. For a binary random variable $X$, there exists no $p_{X}(1) \in \mathbb{R}$ with $0<p_{X}(1)<1 / 2$ for which Equation (7) is fulfilled for $\sigma_{\alpha}$ and $\sigma_{\beta}$. Consequently, $D(S \| \hat{S})$ cannot be zero and by Equation (9), the redundancy $R_{v}(X)$ cannot be zero, either.

Proof. Theorem 8.3 requires $p_{X}(1)>0$, which is always fulfilled for Equations (29) and (30), and $p_{X}(1)<1 / 2$, which yields

$$
\begin{array}{rll}
2^{-\frac{c}{a}}<\frac{1}{2} & \Longleftrightarrow & \frac{c}{a}>1 \\
1-2^{-\frac{d}{b}}<\frac{1}{2} & \Longleftrightarrow & \frac{d}{b}<1 .
\end{array}
$$

Substituting Equation (29) into Equation (30) yields

$$
\begin{array}{r}
2^{-\frac{d}{b}}+2^{-\frac{c}{a}}=1 \\
\Longleftrightarrow \sqrt[a b]{2^{-a d}}+\sqrt[a b]{2^{-b c}}=1 .
\end{array}
$$

From Lemma 8.2 follows that the sum on the left-hand side of Equation (32) cannot become 1 if at least one of the roots is irrational. Since $d / b<1$ by Equation (31), $2^{-d / b}$ must be irrational. Consequently, Equation (32) has no solution for $0<p_{X}(1)<1 / 2$ and the theorem is proven.

\section{CONCLUSION}

Several properties of $\mathrm{V} 2 \mathrm{~V}$ codes are studied for binary i.i.d. sources and strategies for finding minimum redundancy codes are discussed. By analyzing the average code length function of a $\mathrm{V} 2 \mathrm{~V}$ code, a canonical representation for source trees, for code trees, and, consequently, for V2V codes is defined. The prefix merge algorithm allows us to construct a source tree from a canonical representation. It is based on merging particular source words by their prefix. Another important observation is that $\mathrm{V} 2 \mathrm{~V}$ codes can be composed of other $\mathrm{V} 2 \mathrm{~V}$ codes, which leads to the concept of composite and prime $\mathrm{V} 2 \mathrm{~V}$ codes. If a $\mathrm{V} 2 \mathrm{~V}$ code is composite, then its average code length lies between the average code lengths of the V2V codes it is composed of. This property can be useful for finding minimum redundancy V2V codes. For example, composite V2V codes can be removed from a candidate set when the optimal codes are known to be prime, which is often the case.

Various types of size-limited V2V codes are described by defining finite subsets of the infinite set of all possible V2V codes. Minimum redundancy V2V codes are then found by an exhaustive search over the subsets. It is shown how Huffman's algorithm, the package merge algorithm, the concept of canonical V2V codes, and the concept of prime and composite V2V codes can be employed to greatly reduce the complexity of the exhaustive search. The redundancy of selected V2V codes is compared and discussed.

Finally, it is shown that the redundancy of a V2V code cannot be zero for a binary i.i.d. source except for the case where the probability of both binary source symbols is equal to 0.5 . 


\section{ACKNOWLEDGMENTS}

The authors thank Jonathan Pfaff for reviewing the manuscript and for providing many valuable comments.

\section{REFERENCES}

[1] J. Abrahams. 1997. Code and parse trees for lossless source encoding. In Compression and Complexity of Sequences. 145-171. DOI : http://dx.doi.org/10.1109/SEQUEN.1997.666911

[2] I. Boreico. 2008. Linear independence of radicals. Harv. Coll. Math. Rev. 2, 1 (2008), 87-92.

[3] R. R. Coifman and M. V. Wickerhauser. 1992. Entropy-based algorithms for best basis selection. IEEE Trans. Inf. Theory 38, 2 (1992), 713-718. DOI : http://dx.doi.org/10.1109/18.119732

[4] J. B. Connell. 1973. A huffman-shannon-fano code. Proc. IEEE 61, 7 (Jul. 1973), 1046-1047. DOI : http://dx.doi.org/10. 1109/PROC.1973.9200

[5] T. Cover and J. A. Thomas. 1991. Elements of Information Theory. John Wiley \& Sons, Inc. DOI : http://dx.doi.org/10. $1002 / 0471200611$

[6] F. Fabris. 1992. Variable-length-to-variable length source coding: A greedy step-by-step algorithm. IEEE Trans. Inf. Theory 38, 5 (1992), 1609-1617. DOI : http://dx.doi.org/10.1109/18.149517

[7] G. H. Freeman. 1991. Asymptotic convergence of dual-tree entropy codes. In Proceedings of the Data Compression Conference D(CC'91). 208-217. DOI : http://dx.doi.org/10.1109/DCC.1991.213360

[8] G. H. Freeman. 1993. Divergence and the construction of variable-to-variable-length lossless codes by source-word extensions. In Proceedings of the Data Compression Conference (DCC'93). 79-88. DOI : http://dx.doi.org/10.1109/DCC. 1993.253142

[9] S. B. Guthery. 2010. A Motifof Mathematics: History and Application of the Mediant and the Farey Sequence. CreateSpace Independent Publishing Platform.

[10] D. S. Hirschberg and D. A. Lelewer. 1990. Efficient decoding of prefix codes. Commun. ACM 33, 4 (Apr. 1990), 449-459. DOI : http://dx.doi.org/10.1145/77556.77566

[11] D. A. Huffman. 1952. A method for the construction of minimum-redundancy codes. Proc. IRE 40, 9 (1952), $1098-1101$. DOI : http://dx.doi.org/10.1109/JRPROC.1952.273898

[12] F. Jelinek and K. Schneider. 1972. On variable-length-to-block coding. IEEE Trans. Inf. Theory 18, 6 (Nov. 1972), $765-$ 774. DOI : http://dx.doi.org/10.1109/TIT.1972.1054899

[13] H. Kirchhoffer. 2016. Design and Application of Variable-to-variable Length Codes. Ph.D. Dissertation. University of Rostock.

[14] L. G. Kraft. 1949. A Device for Quantizing, Grouping, and Coding Amplitude-modulated Pulses. Master's thesis. Massachusetts Institute of Technology.

[15] L. L. Larmore and D. S. Hirschberg. 1990. A fast algorithm for optimal length-limited Huffman codes. F. ACM 37, 3 (Jul. 1990), 464-473. DOI : http://dx.doi.org/10.1145/79147.79150

[16] D. Marpe, H. Schwarz, and T. Wiegand. 2010. Entropy coding in video compression using probability interval partitioning. In Proceedings of the Picture Coding Symposium (PCS'10). 66-69. DOI: http://dx.doi.org/10.1109/PCS.2010. 5702580

[17] D. Marpe, H. Schwarz, T. Wiegand, and H. Kirchhoffer. 2014. Entropy coding. US Patent 8,907,823. (December 9 2014).

[18] J. G. Michaels and K. H. Rosen. 1991. Applications of Discrete Mathematics. McGraw-Hill, Inc.

[19] J. J. Rissanen. 1976. Generalized kraft inequality and arithmetic coding. IBM J. Res. Dev. 20, 3 (May 1976), $198-203$. DOI : http://dx.doi.org/10.1147/rd.203.0198

[20] E. S. Schwartz and B. Kallick. 1964. Generating a canonical prefix encoding. Commun. ACM 7, 3 (Mar. 1964), 166-169. DOI : http://dx.doi.org/10.1145/363958.363991

[21] P. R. Stubley. 1992. Adaptive Data Compression Using Tree Codes. Ph.D. Dissertation. University of Waterloo.

[22] P. R. Stubley. 1994. Adaptive variable-to-variable length codes. In Proceedings of the Data Compression Conference (DCC'94). 98-105. DOI : http://dx.doi.org/10.1109/DCC.1994.305917

[23] P. R. Stubley. 1994. On the redundancy of optimum fixed-to-variable length codes. In Proceedings of the Data Compression Conference (DCC'94). 90-97. DOI : http://dx.doi.org/10.1109/DCC.1994.305916

[24] B. P. Tunstall. 1967. Synthesis of Noiseless Compression Codes. Ph.D. Dissertation. Georgia Institute of Technology.

[25] T. Wiegand and H. Schwarz. 2011. Source Coding: Part I of Fundamentals of Source and Video Coding. Now Publishers Inc. DOI : http://dx.doi.org/10.1561/2000000010

Received November 2017; revised May 2018; accepted May 2018 